\title{
Stereotactic Vacuum-Assisted Breast Biopsy in Ductal Carcinoma in situ: Residual Microcalcifications and Intraoperative Findings
}

\author{
Benedict Krischer ${ }^{\mathrm{a}}$ Serafino Forte ${ }^{\mathrm{c}}$ Gad Singer $^{\mathrm{b}}$ Rahel A. Kubik-Huch ${ }^{\mathrm{c}}$ \\ Cornelia Leo ${ }^{a}$ \\ ${ }^{a}$ Department of Gynecology, Breast Center, Kantonsspital Baden, Baden, Switzerland; ${ }^{\mathrm{b}}$ Department of Pathology,

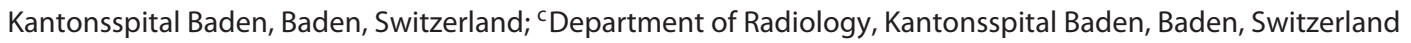

\author{
Keywords \\ Ductal carcinoma in situ · Premalignant lesion . \\ Overtreatment $\cdot$ Breast cancer
}

\begin{abstract}
Purpose: The question of overtreatment of ductal carcinoma in situ (DCIS) was raised because a significant proportion of especially low-grade DCIS lesions never progress to invasive cancer. The rationale for the present study was to analyze the value of stereotactic vacuum-assisted biopsy (VAB) for complete removal of DCIS, focusing on the relationship between the absence of residual microcalcifications after stereotactic $V A B$ and the histopathological diagnosis of the definitive surgical specimen. Patients and Methods: Data of 58 consecutive patients diagnosed with DCIS by stereotactic VAB in a single breast center between 2012 and 2017 were analyzed. Patient records from the hospital information system were retrieved, and mammogram reports and images as well as histopathology reports were evaluated. The extent of microcalcifications before and after biopsy as well as the occurrence of DCIS in biopsy and definitive surgical specimens were analyzed and correlated. Results: There was no correlation between the absence of residual microcalcifications in the post-biopsy mammogram and the absence of residual DCIS in the final surgical specimen $(p=0.085)$. Upstaging to invasive cancer was recorded in 4 cases (13\%) but occurred only in the group that had high-grade DCIS on biopsy. Lowgrade DCIS was never upgraded to high-grade DCIS in the definitive specimen. Conclusions: The radiological absence of microcalcifications after stereotactic biopsy does not rule
\end{abstract}

out residual DCIS in the final surgical specimen. Since upstaging to invasive cancer is seen in a substantial proportion of high-grade DCIS, the surgical excision of high-grade DCIS should remain the treatment of choice.

() 2019 S. Karger AG, Basel

\section{Introduction}

Since the introduction of breast cancer screening programs, ductal carcinoma in situ (DCIS) shows an increasing incidence. Suspicious microcalcifications on mammography prompt a tissue biopsy, which is usually performed using the stereotactic vacuum-assisted biopsy (VAB) method [1]. VAB has been found to have a higher diagnostic accuracy than core needle biopsies [2]. The preoperative histopathological diagnosis determines the consecutive surgical management, especially regarding axillary staging [1]. VAB may completely remove microcalcifications seen by mammography [3], in particular lesions measuring up to $10 \mathrm{~mm}$ [4]. However, when the final surgical specimen is examined histopathologically, up to $70 \%$ of these patients will show tumor lesions $[3,4]$.

In $5-15 \%$ of the patients, an upstaging occurs, with an initial diagnosis of DCIS on VAB but a later detection of invasive carcinoma in the excised tissue $[4,5]$. Such an upstaging is consequential for the patients, since DCIS requires a different diagnostic workup than an invasive carcinoma [1]. A high level of diagnostic accuracy is

R.A.K.-H. and L.C. contributed equally to this work. 


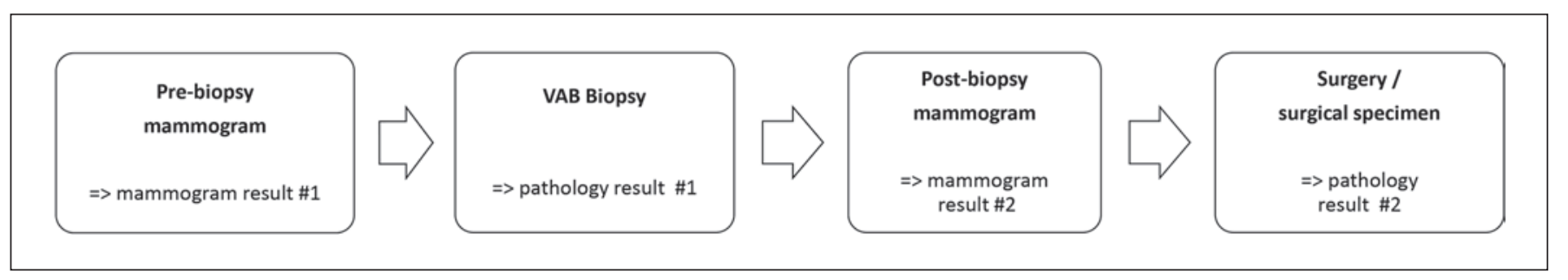

Fig. 1. Patient flow and data generated.

Table 1. Classification of post-biopsy mammograms

\begin{tabular}{ll}
\hline $\begin{array}{l}\text { Mammogram \#2 } \\
\text { classification }\end{array}$ & Findings \\
\hline 0 & No microcalcifications visible after VAB \\
1 & Solitary microcalcifications after VAB \\
2 & Extensive microcalcifications after VAB \\
3 & Multifocal microcalcifications after VAB \\
\hline
\end{tabular}

therefore needed to avoid additional procedures for the patients.

To our knowledge, it has not previously been determined how often - despite complete removal of microcalcifications in low-grade DCIS by VAB - residual low-grade DCIS remains in the surgical specimen. In our study, we therefore aimed to investigate - in pure DCIS - the association between the absence of residual microcalcifications after stereotactic VAB and the histological result in the definitive surgical specimen. Moreover, we assessed how often, in this setting, upgrading to high-grade DCIS or upstaging to invasive breast cancer occurred.

The present study contributes to the ongoing research investigating a possible role for $\mathrm{VAB}$ in the complete removal of DCIS, as well as the tendency of VAB to cause understaging in DCIS.

\section{Patients, Materials, and Methods}

\section{Selection of Patients}

After approval, hospital records were reviewed. The data of all patients diagnosed with DCIS by stereotactic VAB in our center between 2012 and 2017 were retrieved. Patient flow and data generation points are shown in Figure 1.

All patients underwent VAB for the evaluation of mammographically detected microcalcifications not visible by sonography (Fig. 1, mammogram \#1). The microcalcifications were evaluated and classified according to the BIRADS classification system (ACR BI-RADS Atlas ${ }^{\circledR} 5$ th Edition). VAB was performed by radiologists specifically trained in $\mathrm{VAB}$ using the Mammotome Revolve on a Fisher table with 7- or 10-gauge needles, depending on the size and localization of microcalcifications (Mammotome, Cincinnati, $\mathrm{OH}$, USA; 7 or 10 gauge). The procedure was standardized according to accredited protocols to ensure quality control $[6,7]$. The initial histopathological diagnosis of DCIS (pathology result \#1) was followed by an open surgical excision with a definitive histopathological examination (pathology result \#2). Preoperatively, the lesion was stereotactically marked using a wire. Before this procedure, another mammography was performed in cranio-caudal and mediolateral oblique views with the full-field digital technique (mammogram result \#2) to assess residual microcalcifications and to guide placement of the wire. The definitive therapy was guided by the final histopathological result.

\section{Evaluation of Post-Biopsy Microcalcifications}

All records obtained after initial database screening were reviewed, including evaluation of all pre- and post-biopsy mammograms by a radiologist and a gynecologist from the study team. The pre-biopsy mammogram (\#1) was evaluated for the presence of microcalcifications, their localization, and their maximum extent. The post-biopsy mammogram result (\#2) was classified according to Table 1 . The pathology reports were independently reviewed by two team members. The DCIS grades in the biopsy and in the final tissue sample were recorded.

\section{Statistical Analysis}

To test the association between residual microcalcifications after biopsy and the presence of residual DCIS in the surgical specimen, Cohen's kappa coefficient was used, together with the frequency table. The same coefficient was calculated to measure the association between microcalcification after biopsy and residual DCIS in the final surgical specimen, analyzing DCIS grade 1 and DCIS grades $2 / 3$ separately. The significance of the association between DCIS grade and the residual DCIS in the final surgical specimen without microcalcification after biopsy was assessed using Fisher's exact test.

A shift table of DCIS grade in the biopsy and surgical specimen, respectively, was produced to show how DCIS grade varied after surgery. Binomial testing of upgrading to grade 3 in the surgical specimen was also conducted to determine whether upgrading might occur by chance alone. The same analyses were also undertaken to test for upstaging to invasive cancer. Bar charts were used to display the proportion of DCIS grade, residual microcalcification, and residual DCIS without microcalcifications.

\section{Results}

\section{Patients}

After initial database screening, 61 patients were included in the study. In the data review process, 3 patients had to be excluded. The reason for this exclusion were incomplete patient records; e.g., if surgery was performed in a different hospital and therefore histopathology re- 


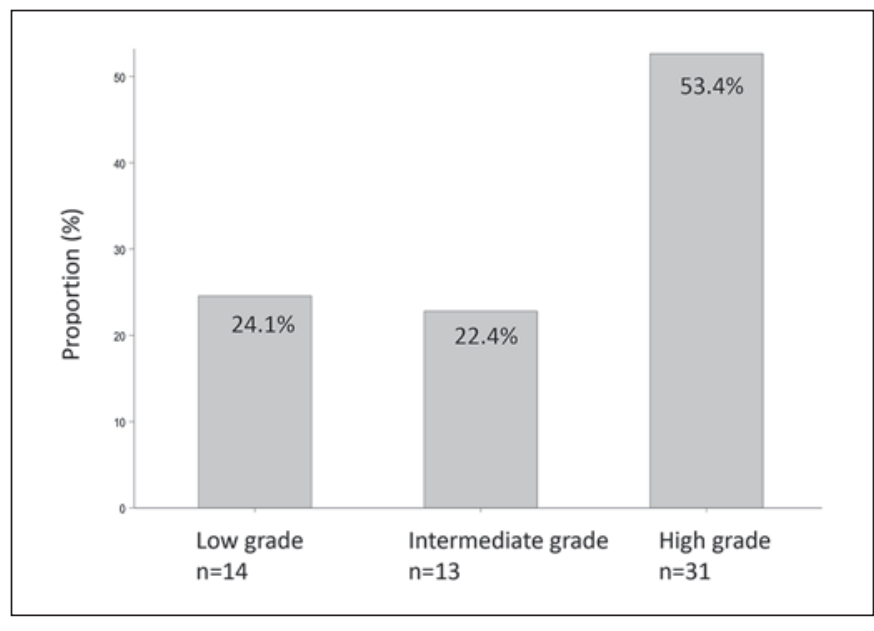

Fig. 2. Distribution of DCIS grade in a biopsy specimen.

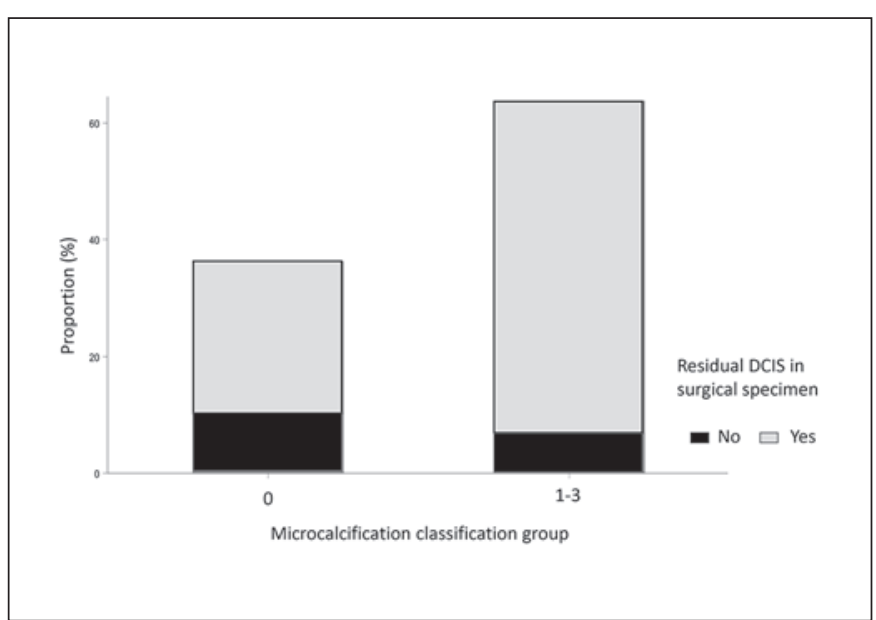

Fig. 3. Cases by microcalcification group according to post-biopsy mammogram and residual DCIS in the final surgery specimen.

Table 2. Residual DCIS in the surgical specimen given no microcalcification after biopsy versus DCIS grade after biopsy

\begin{tabular}{|c|c|c|c|}
\hline & \multicolumn{2}{|c|}{$\begin{array}{l}\text { Classification group } 0 \text { (no microcalcifications detectable in post-biopsy } \\
\text { mammogram) DCIS grade after biopsy }\end{array}$} & \multirow[t]{2}{*}{$\begin{array}{l}\text { Total } \\
(n=21)\end{array}$} \\
\hline & $\begin{array}{l}\text { grade } 1 \text { (low grade) } \\
(n=7)\end{array}$ & $\begin{array}{l}\text { grades } 2 / 3 \text { (intermediate and high } \\
\text { grade) }(n=14)\end{array}$ & \\
\hline \multicolumn{4}{|c|}{ Residual DCIS in surgical specimen } \\
\hline No & $4(57.1 \%)$ & $2(14.3 \%)$ & $6(28.6 \%)$ \\
\hline Yes & $3(42.9 \%)$ & $12(85.7 \%)$ & $15(71.4 \%)$ \\
\hline
\end{tabular}

sults or images were not available. Therefore, 58 patients were included in the data analysis.

\section{Patient and Radiological Characteristics}

The patients' age ranged from 37 to 81 years with a median of 59 years. The dimensions of microcalcification areas ranged from $2 \times 3 \times 4 \mathrm{~mm}$ to $23 \times 68 \times 39 \mathrm{~mm}$.

In the post-biopsy mammogram, 21 cases $(36.2 \%)$ showed no residual microcalcifications (classification group 0 ). In 10 cases $(17.2 \%)$, only solitary residual microcalcifications (group 1) were recorded within the targeted microcalcification area. In 18 cases (31.0\%), extensive residual microcalcifications were detected in the targeted region (>50\%) (group 2), and in 9 cases $(15.5 \%)$, there were multifocal microcalcifications outside the targeted microcalcification region (group 3 ).

\section{Histopathological Characteristics}

The distribution of low-grade (14 cases), intermediate-grade (13 cases), and high-grade DCIS (31 cases) after $\mathrm{VAB}$ is shown in Figure 2.

All patients underwent surgery after the diagnosis of DCIS. Fifty-four patients received breast-conserving surgery, the other 4 were treated by mastectomy. In the final surgical specimen, 10 cases (17.2\%) had no residual DCIS. In 44 cases (75.9\%), residual DCIS was detected, and in 4 cases $(6.9 \%)$, there was an upstaging to invasive cancer.

Association between DCIS Grade in the Biopsy Sample and Pathological Findings in the Surgical Specimen

Of the 21 cases without radiologically detectable residual microcalcifications, 15 (71.4\%) showed residual DCIS in the final surgery specimen, whereas in 6 cases $(28.6 \%)$, no DCIS residues were detected (Fig. 2). In addition, of the 37 cases with radiologically detected residues after $\mathrm{VAB}$, 4 cases $(10.8 \%)$ had no histopathological DCIS residues in the surgical specimen (Fig. 3). There was no correlation between post-biopsy absence of microcalcifications in the mammogram and absence of residual DCIS in the final surgical specimen $(p=0.085)$. Moreover, there was no association between DCIS grade and presence of DCIS residues in these cases (Table 2, $p=0.120$ ).

Four cases diagnosed as DCIS upon biopsy were upstaged to invasive cancer following open excision. All of these cases were high-grade lesions and represented $13.3 \%$ of all high-grade lesions. All these cases had shown significant residual microcalcifications on the post-biopsy mammogram (Fig. 4, 5a-d, 6A, B). 
Fig. 4. Cases by microcalcification group, residual DCIS after biopsy, and upgrading to invasive cancer in the surgical specimen.
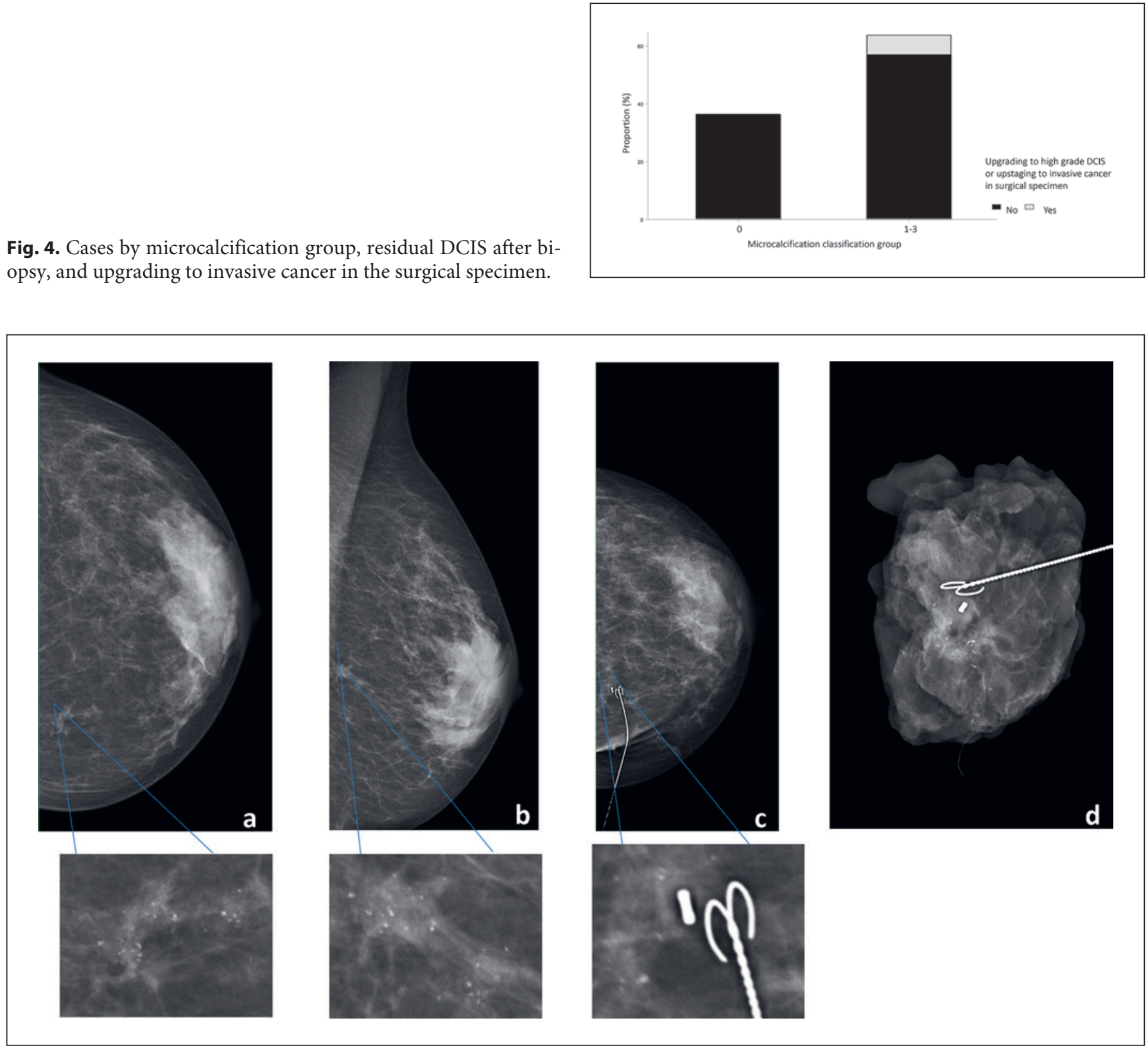

Fig. 5. a Mammogram in the cranio-caudal view showing a group of microcalcifications (enlarged in the Inset). b The same patient with a mammogram in the medio-lateral-oblique view (microcalcifications enlarged in the Inset). c Post-biopsy mammogram (in the craniocaudal view), the residual microcalcifications categorized as "group 2" (extensive residual microcalcifications after VAB). A wire is placed to guide surgery (Inset). d Intraoperative radiography of the surgical specimen.

An upgrade from low- or intermediate-grade DCIS to high-grade DCIS was not recorded (Table 3).

\section{Discussion}

We investigated whether radiologically confirmed complete removal of DCIS-associated microcalcifications via stereotactic $\mathrm{VAB}$ is predictive of the absence of residual DCIS in the final surgical specimen. In this study, we show that even with complete removal of microcalci- fications seen on the post-biopsy mammogram, DCIS residues can be found in the definitive surgical specimen. This was independent of DCIS grade. A caveat of our study is the monocentric nature of it and the limited number of 61 cases of DCIS.

DCIS is usually not associated with a palpable mass [8]. Today, it is typically diagnosed as nonpalpable microcalcifications in screening mammograms [9]. If they do not represent benign lesions, microcalcifications on mammography can be associated with DCIS or even (micro-) invasive carcinoma [10]. Microcalcifications are classi- 

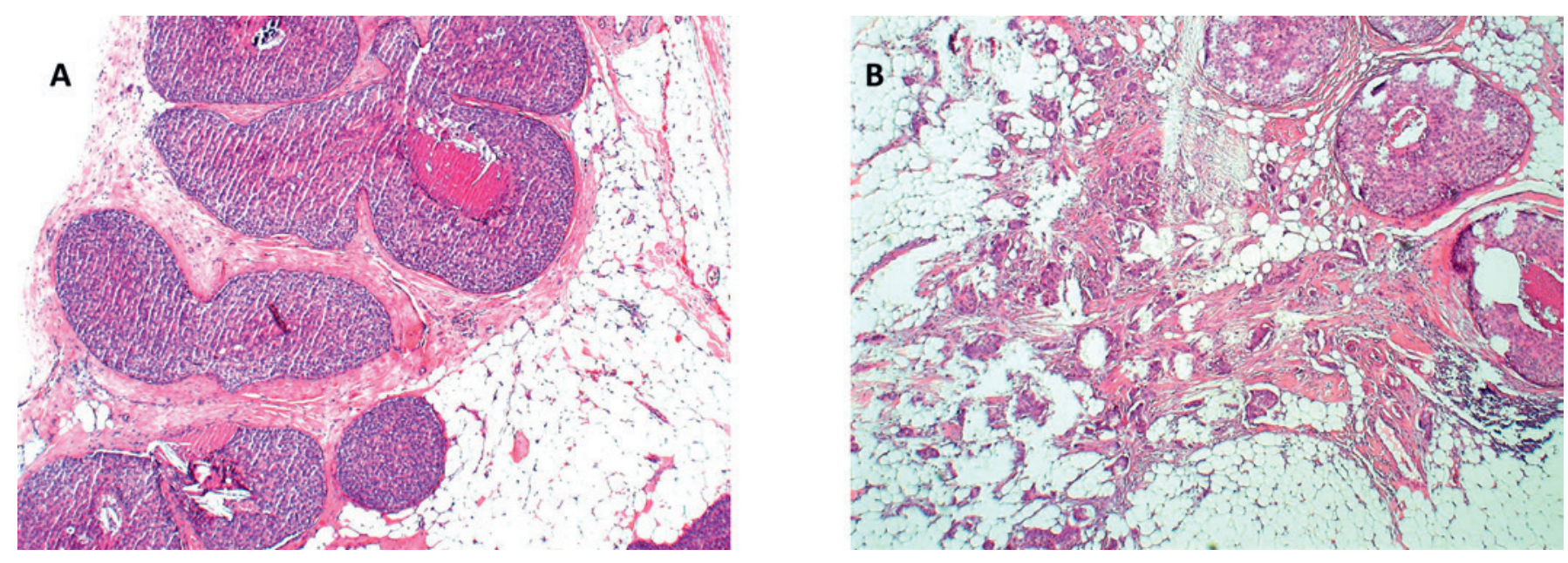

Fig. 6. A Microscopic image of the initial biopsy showing high-grade DCIS with focal comedo necrosis $(\times 250$ magnification). B Microscopic image of the biopsy after final surgery showing high-grade DCIS with focal comedo necrosis (on the right) and invasive ductal carcinoma (center and left, $\times 250$ magnification). The histologies represent the same case as the mammograms in Figure 5.

Table 3. Shift table of DCIS grade after VAB versus DCIS grade in the surgical specimen

\begin{tabular}{lllllll}
\hline \multicolumn{7}{l}{ DCIS grade in surgical specimen } \\
\cline { 2 - 7 } & $\begin{array}{l}\text { no residual DCIS } \\
(n=8)\end{array}$ & $\begin{array}{l}\text { low } \\
(n=7)\end{array}$ & $\begin{array}{l}\text { intermediate } \\
(n=15)\end{array}$ & $\begin{array}{l}\text { high } \\
(n=26)\end{array}$ & $\begin{array}{l}\text { missing } \\
(n=2)\end{array}$ & $\begin{array}{l}\text { total } \\
(n=58)\end{array}$ \\
\hline $\begin{array}{l}\text { DCIS grade after biopsy } \\
\text { Low }\end{array}$ & $5(62.5 \%)$ & $7(100 \%)$ & $1(6.7 \%)$ & 0 & $1(50 \%)$ & $14(24.1 \%)$ \\
Intermediate & $2(25.0 \%)$ & 0 & $11(73.3 \%)$ & 0 & 0 & $13(22.4 \%)$ \\
High & $1(12.5 \%)$ & 0 & $2(13.3 \%)$ & $26(100 \%)$ & $1(50 \%)$ & $30(51.7 \%)$ \\
Missing & 0 & 0 & $1(6.7 \%)$ & 0 & 0 & $1(1.7 \%)$ \\
\hline
\end{tabular}

fied according to the BIRADS classification system (ACR BI-RADS Atlas 5th edition). To categorize calcifications, their characteristics (e.g., amorphous, fine pleomorphic, or fine-linear branching) as well as their distribution in the breast (e.g., diffuse, regional, grouped, linear, and segmental) are taken into consideration. As shown by Müller-Schimpfle et al. [11] and confirmed by Kaltenbach et al. [12], combining both, the morphology and distribution characteristics of microcalcifications, leads to improved accuracy in estimating malignancy.

As the size of the microcalcification area increases, so does the risk of invasive disease [1]. A size of $\geq 11 \mathrm{~mm}$ was found to be associated with an increased risk of invasive carcinoma [10]. This is in line with our findings that upstaging to invasive cancer exclusively occurred in cases with extensive microcalcifications. There was an upgrade to invasive cancer in 4 cases, which corresponds to $6.9 \%$ of the whole cohort. All of these cases showed high-grade DCIS in the biopsy specimen. Therefore, $13.3 \%$ of the DCIS that were high-grade upon biopsy showed invasive cancer in the final surgical specimen, whereas no low- or intermediate-grade DCIS cases on biopsy had to be up- staged. This is in line with the study by Soumian et al. [13] who showed that $23 \%$ of the high-grade DCIS by VAB histopathology were upstaged to invasive carcinoma after open excision, while there was no upstaging in the cases with asymptomatic low-grade DCIS. A recent study by Grimm et al. [14] showed an overall rate of upstaging to invasive disease of $17 \%$. In their study, there were fewer cases of upstaging in the group with non-high grade DCIS, corresponding to a frequency between 6 and $10 \%$.

The existing literature about the natural course of DCIS suggests that $>50 \%$ of the high-grade DCIS lesions will ultimately develop into invasive breast carcinoma within 5 years. By contrast, only $35-50 \%$ of the low-grade DCIS appear to develop into invasive cancer over an extended period of $>40$ years [15]. In a recent publication, Maxwell et al. [16] described that the cumulative incidence of invasion was significantly higher in high-grade DCIS compared to other grades. They found that $48 \%$ of the high-grade DCIS developed into invasive cancer over a median interval of 38 months, whereas only $18 \%$ of the low-grade DCIS progressed to invasive carcinoma over a median time of 51 months. 
As of today, approximately $20 \%$ of all breast neoplasms are found to be DCIS lesions. Since DCIS is regarded as a preinvasive lesion that may lead to invasive breast cancer, its local treatment is equally aggressive. Treatment usually consists of surgical excision and - in case of breastconserving surgery - of additional adjuvant radiotherapy. Radiotherapy was shown to reduce the risk of local recurrence by half independently of DCIS grade, but this did not translate into differences in the overall survival [17, 18].

However, over the past years, the question of overdiagnosis and, hence, overtreatment of DCIS has arisen. In particular, low-grade DCIS appears to be only a risk factor for developing invasive cancer rather than an obligatory precancerous lesion [19]. In our study, there was no case of upstaging of low- or intermediate-grade DCIS to invasive cancer. This raises the question whether - in selected cases - observation rather than surgery could be an option. Given the unclear long-term natural history of non-high-grade DCIS - especially low-grade DCIS - the strategy of active surveillance of these women needs to be investigated in large prospective clinical trials. Such clinical trials are ongoing (e.g., LORIS [20], LORD [21]).

In conclusion, the complete removal of microcalcifications with stereotactic biopsy does not rule out residual DCIS in the final surgical specimen. Since upstaging to invasive cancer occurs in a substantial proportion of high-grade DCIS, complete surgical excision of highgrade DCIS remains the treatment of choice.

\section{Statement of Ethics}

This study was approved by the ethics committee (Ethikkommission Nordwest- und Zentralschweiz, EKNZ, ID 2018-00625). Since retrospective anonymized data were used, no individual patient informed consent had to be obtained, which was in agreement with the ethics committee.

\section{Disclosure Statement}

The authors declare that they have no conflicts of interest to disclose.

\section{References}

1 O’Flynn EA, Morel JC, Gonzalez J, Dutt N, Evans D, Wasan R, et al. Prediction of the presence of invasive disease from the measurement of extent of malignant microcalcification on mammography and ductal carcinoma in situ grade at core biopsy. Clin Radiol. 2009 Feb;64(2):178-83.

2 Kumaroswamy V, Liston J, Shaaban AM. Vacuum assisted stereotactic guided mammotome biopsies in the management of screen detected microcalcifications: experience of a large breast screening centre. J Clin Pathol. 2008 Jun;61(6):766-9.

3 Penco S, Rizzo S, Bozzini AC, Latronico A, Menna S, Cassano E, et al. Stereotactic vacuum-assisted breast biopsy is not a therapeutic procedure even when all mammographically found calcifications are removed: analysis of 4,086 procedures. AJR Am J Roentgenol. 2010 Nov;195(5):1255-60.

4 Kettritz U, Rotter K, Schreer I, Murauer M, Schulz-Wendtland R, Peter D, et al. Stereotactic vacuum-assisted breast biopsy in 2874 patients: a multicenter study. Cancer. 2004 Jan; 100(2):245-51.

5 Brem RF, Schoonjans JM, Sanow L, Gatewood OM. Reliability of histologic diagnosis of breast cancer with stereotactic vacuum-assisted biopsy. Am Surg. 2001 Apr;67(4):388-92.

6 Saladin C, Haueisen H, Kampmann G, Oehlschlegel C, Seifert B, Rageth L, et al.; MIBB Group. Lesions with unclear malignant potential (B3) after minimally invasive breast biopsy: evaluation of vacuum biopsies performed in Switzerland and recommended further management. Acta Radiol. 2016 Jul; 57(7):815-21

7 Imschweiler T, Haueisen H, Kampmann G, Rageth L, Seifert B, Rageth C, et al. MRI-guid- ed vacuum-assisted breast biopsy: comparison with stereotactically guided and ultrasound-guided techniques. Eur Radiol. 2014 Jan;24(1):128-35.

8 Bijker N, Donker M, Wesseling J, den Heeten GJ, Rutgers EJ. Is DCIS breast cancer, and how do I treat it? [Review]. Curr Treat Options Oncol. 2013 Mar;14(1):75-87.

9 Brown AP. Ductal carcinoma in situ of the breast. N Engl J Med. 2004 Jul;351(4):399-402.

10 Stomper PC, Geradts J, Edge SB, Levine EG. Mammographic predictors of the presence and size of invasive carcinomas associated with malignant microcalcification lesions without a mass. AJR Am J Roentgenol. 2003 Dec;181(6):1679-84.

11 Müller-Schimpfle M, Wersebe A, Xydeas T, Fischmann A, Vogel U, Fersis N, et al. Microcalcifications of the breast: how does radiologic classification correlate with histology? Acta Radiol. 2005 Dec;46(8):774-81.

12 Kaltenbach B, Brandenbusch V, Möbus V, Mall G, Falk S, van den Bergh M, et al. A matrix of morphology and distribution of calcifications in the breast: analysis of 849 vacuum-assisted biopsies. Eur J Radiol. 2017 Jan; 86:221-6.

13 Soumian S, Verghese ET, Booth M, Sharma $\mathrm{N}$, Chaudhri S, Bradley S, et al. Concordance between vacuum assisted biopsy and postoperative histology: implications for the proposed Low Risk DCIS Trial (LORIS). Eur J Surg Oncol. 2013 Dec;39(12):1337-40.

14 Grimm LJ, Ryser MD, Partridge AH, Thompson AM, Thomas JS, Wesseling J, et al. Surgical Upstaging Rates for Vacuum Assisted Biopsy Proven DCIS: Implications for Active Surveillance Trials. Ann Surg Oncol. 2017 Nov;24(12):3534-40.
15 Sanders ME, Schuyler PA, Simpson JF, Page DL, Dupont WD. Continued observation of the natural history of low-grade ductal carcinoma in situ reaffirms proclivity for local recurrence even after more than 30 years of follow-up. Mod Pathol. 2015 May;28(5):6629.

16 Maxwell AJ, Clements K, Hilton B, Dodwell DJ, Evans A, Kearins O, et al.; Sloane Project Steering Group. Risk factors for the development of invasive cancer in unresected ductal carcinoma in situ. Eur J Surg Oncol. 2018 Apr; 44(4):429-35.

17 Donker M, Litière S, Werutsky G, Julien JP, Fentiman IS, Agresti R, et al. Breast-conserving treatment with or without radiotherapy in ductal carcinoma In Situ: 15-year recurrence rates and outcome after a recurrence, from the EORTC 10853 randomized phase III trial. J Clin Oncol. 2013 Nov;31(32):4054-9.

18 Narod SA, Iqbal J, Giannakeas V, Sopik V, Sun P. Breast Cancer Mortality After a Diagnosis of Ductal Carcinoma In Situ. JAMA Oncol. 2015 Oct;1(7):888-96.

19 Esserman L, Yau C. Rethinking the Standard for Ductal Carcinoma In Situ Treatment. JAMA Oncol. 2015 Oct;1(7):881-3.

20 Francis A, Fallowfield L, Rea D. The LORIS Trial: addressing overtreatment of ductal carcinoma in situ. Clin Oncol (R Coll Radiol). 2015 Jan;27(1):6-8.

21 Elshof LE, Tryfonidis K, Slaets L, van Leeuwen-Stok AE, Skinner VP, Dif N, et al. Feasibility of a prospective, randomised, open-label, international multicentre, phase III, noninferiority trial to assess the safety of active surveillance for low risk ductal carcinoma in situ - The LORD study. Eur J Cancer. 2015 Aug;51(12):1497-510. 